\title{
Update on Antifungal Drug Resistance
}

\author{
David S. Perlin ${ }^{1} \cdot$ Erika Shor $^{1} \cdot$ Yanan Zhao $^{1}$
}

Published online: 22 April 2015

(C) Springer International Publishing AG 2015

\begin{abstract}
Invasive fungal infections remain a major source of global morbidity and mortality, especially among patients with underlying immune suppression. Successful patient management requires antifungal therapy. Yet, treatment choices are restricted due to limited classes of antifungal agents and the emergence of antifungal drug resistance. In some settings, the evolution of multidrug-resistant strains insensitive to several classes of antifungal agents is a major concern. The resistance mechanisms responsible for acquired resistance are well characterized and include changes in drug target affinity and abundance, and reduction in the intracellular level of drug by biofilms and efflux pumps. The development of high-level and multidrug resistance occurs through a stepwise evolution of diverse mechanisms. The genetic factors that influence these mechanisms are emerging and they form a complex symphony of cellular interactions that enable the cell to adapt and/or overcome drug-induced stress. Drivers of resistance involve a complex blend of host and microbial factors. Understanding these mechanisms will facilitate development of better diagnostics and therapeutic strategies to overcome and prevent antifungal resistance.
\end{abstract}

Keywords Antifungal resistance - Acquired resistance · Candidaalbicans Candidaglabrata $\cdot$ Aspergillusfumigatus . Azoles $\cdot$ Echinocandins $\cdot$ Polyenes

This article is part of the Topical Collection on Mycology

David S. Perlin

perlinds@njms.rutgers.edu

Erika Shor

es783@njms.rutgers.edu

Yanan Zhao

zhaoy1@njms.rutgers.edu

1 Public Health Research Institute, Rutgers Biomedical and Health Sciences, New Jersey Medical School, 185 S Orange Ave, Newark, NJ 07103, USA

\section{Introduction}

Serious fungal infections afflict millions of patients annually resulting in more than $1,350,000$ deaths [1]. The most serious fungal infections occur as a consequence of other serious health problems such as asthma, AIDS, cancer, and organ transplantation, and they all require antifungal therapy for a successful outcome. Failure to treat effectively either because of diagnostic delays or missed diagnosis often leads to death or serious illness. This recognition has resulted in a significant increase in antifungal agents use for the treatment and prevention of fungal infections. Yet, therapeutic options are limited, as the most widely used antifungal drugs comprise only a few chemical classes including azoles (fluconazole, voriconazole, posaconazole, and isavuconazole) and polyenes (amphotericin B), which modify the cell membrane, nucleic acids and protein flucytosine (5-fluorocytosine), and the cell wall echinocandins (caspofungin, anidulafungin, and micafungin). Predictably, resistant strains emerge during therapy, and it is a confounding factor for successful clinical outcome as it eliminates important antifungal classes leaving restricted treatment options. Resistance may result from selection of inherently less susceptible strains or from emergence of acquired drug resistance during therapy in otherwise susceptible strains. It is the latter that is the principal subject of this review, although many acquired mechanisms also account for naturally occurring reduced susceptibility of some species. A greater understanding of factors promoting mechanism-specific resistance is important to help overcome resistance emergence.

\section{Epidemiology and Emergence of Multidrug Resistance}

\section{Inherent Resistance Selection}

Resistance to antifungal agents remains relatively uncommon, as the vast majority of fungi retain susceptibility to commonly 
used antifungal agents. In some cases, prominent resistance results from selection of less susceptible species. The azole antifungal agents are the most prominent example of drug selection for less susceptible species [2]. Numerous global epidemiological studies have documented the impact of widespread triazole use on the distribution and shift of Candida species toward less susceptible strains like Candida glabrata and Candida krusei. In many regions where azole use (e.g., fluconazole) is prevalent, there has been a shift away from Candida albicans as the predominant cause of invasive infections toward less susceptible non-C. albicans species [3]. C. glabrata has inherent reduced susceptibility to fluconazole and it is the species whose incidence has increased the most to account for a decrease in the prevalence of $C$. albicans [3, 4]. Similarly, fluconazole use is linked to emergence of the highly resistant C. krusei [5] and Candida guilliermondii [6]. In many cases, inherent resistance in Candida species to fluconazole also carries with resistance to more highly active triazoles like voriconazole. This is not true for Aspergillus and other molds that are resistant to fluconazole but susceptible to more highly active triazoles. Yet, breakthrough infections against highly active triazole drugs have been reported for Aspergillus ustus [7] and Aspergillus fumigatus-like species Aspergillus lentulus, which show pleiotropic resistance to multiple antifungal drugs [8,9]. Sometimes, a susceptible species develops a prevalent variant that is the source of resistant infections. In the bacterial world, the regional and global spread of drug-resistant strains from a common progenitor is commonly observed. Such transmission is not typically observed for fungal drug resistance. A notable exception occurred with the recent emergence of a multidrug-resistant variant of $A$. fumigatus in the Netherlands $[10,11]$. This highly azole-resistant strain variant was selected in the environment as a consequence of the prevalent use of agricultural azoles. The resistance mechanism unique to these isolates will be discussed later, but such resistant strains are spreading through Europe and into parts of Asia [12].

\section{Acquired Resistance}

"Acquired" refers to acquisition (or latent induction) of a resistance mechanism during therapy. It is less common but not an inconsequential event. Growing concerns have been raised about acquired antifungal drug resistance involving azole resistance in A. fumigatus and echinocandin resistance in Candida [13-15]. Azole resistance in A. fumigatus is widespread globally with high geographic variance since the first report of itraconazole resistance in 1997 [16]. In the Netherlands, the prevalence of resistance increased from $2 \%$ in 2000 to $8 \%$ in 2009 predominated by $\mathrm{TR}_{34} / \mathrm{L} 98 \mathrm{H}$, a resistance mechanism which has been considered as environmentally acquired and associated with the use of agricultural fungicides [17]. While $\mathrm{TR}_{34} / \mathrm{L} 98 \mathrm{H}$ along with the newly emerged $\mathrm{TR}_{46} / \mathrm{Y} 121 \mathrm{~F} / \mathrm{T} 289 \mathrm{~A}$ are spreading and widely reported in many other countries [18-23], epidemiological data in the UK demonstrated a more drastic increase of resistance from $5 \%$ in 2004 to $14 \%$ in 2008 and $20 \%$ in 2009 with more versatile (CYP51A and non-CYP51A mediated) underlying mechanisms, which were mainly induced by long-term azole therapy in chronic infection patients [24, 25]. Unlike azole resistance, the frequency of echinocandin resistance remains relatively low $(<2-3 \%)$ with $C$. albicans and most other Candida species [26-29]. However, a notable exception is C. glabrata, where an alarming trend of rising echinocandin resistance poses a serious clinical challenge since many isolates display azole cross-resistance $[30 \bullet, 31,32]$. A recent study of $C$. glabrata bloodstream isolates documented the rising rate of echinocandin resistance from 4.9 to $12.3 \%$ in 2001-2010 [30••]. Of note, resistance rates in C. glabrata varies range from $\sim 3 \%$ to over $10 \%$ in recent surveillance studies, depending on the geographic region, subpopulation, and data collecting method of the study [14, 30••, 31-33] (Fig. 1). Nevertheless, rapid acquisition of resistance during therapy for C. glabrata infection with subsequent unfavorable outcome is worrisome.

\section{Mechanisms of Resistance}

Prominent antifungal resistance mechanisms have been detailed in recent years. The mechanisms generally involve reduced drug uptake, modification of the drug target, and/or a reduction in the cellular level of drug due to upregulation of drug efflux transporters (pumps) and biofilms, which restrict drug entry (Fig. 2). Fungi have evolved a number of genetic regulatory features that induce or promote specific resistance mechanisms.

\section{Biofilms}

Yeasts and molds readily form biofilms [34, 35], which display an organized three-dimensional structure comprised of a dense network of cells in an exopolymeric matrix of carbohydrates, proteins, and nucleic acids. Drug sequestration within the extracellular matrix is the largest determinant of the multidrug resistance phenotype of biofilms [36]. Biofilms restrict access to echinocandin drugs and they are intrinsically resistant to azoles. The mechanisms include drug sequestration and expression of drug efflux transporters [34, 35, 37-39]. Matrix production is highly regulated and is a key resistance factor for Candida species [40]. Biosynthesis of $\beta-1,3$-glucan by glucan synthase is critical to the biofilm resistance properties. Downstream components of the yeast PKC pathway, including SMI1, RLM1, RHO1, and FKS1, regulate $\beta$-1,3-glucan biosynthesis and biofilm matrix production $[36,41-43]$, as 
Fig. 1 Echinocandin resistance in C. glabrata in Europe and America. Resistance rate varies among different studies.

The rate reported from institutional studies is higher than that from population-based surveys, where only the initial blood isolate is included to avoid biasing the data set. Adapted from Arendrup et al. [14]

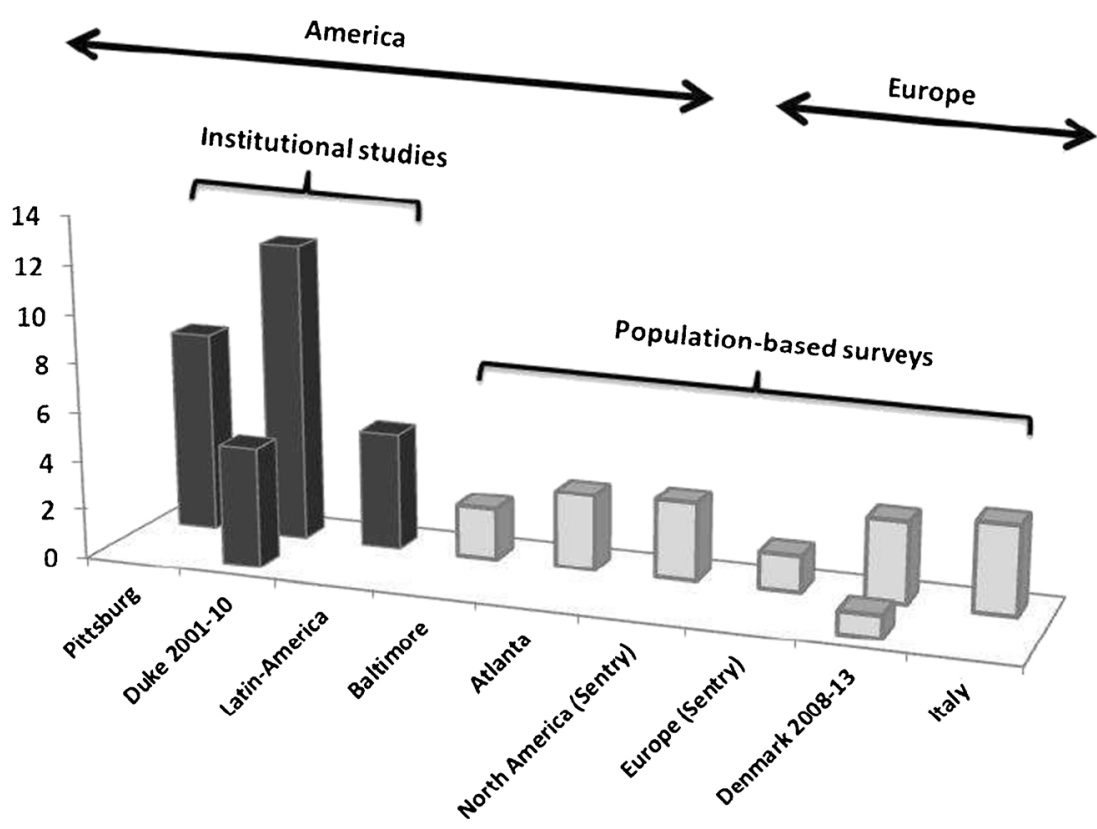

well as other cellular components such as alcohol dehydrogenases Adh5, Csh1, and Ifd6 [44].

\section{Drug Target Modification}

Genetic modification of the drug target resulting in reduced affinity for drug is one of the most prominent mechanisms for antifungal resistance. For echinocandin drugs, target site modification is sufficient to confer resistance, as other mechanisms (e.g., drug pumps) are not associated with clinical resistance [45]. Echinocandins inhibit glucan synthase, which blocks the biosynthesis of the critical cell wall polymer $(1,3)-\beta$-D-glucan. A limited number of mutations in two highly conserved hotspot regions of the FKS genes encoding glucan synthase confer resistance (Table 1). The most prominent mutations in C. albicans associated with clinical failures encode amino acid substitutions at Fks 1 positions Phe641 and Ser645 [46]. These target site modifications decrease the sensitivity of enzyme for drug by as much as several thousand fold [47•, 48, 49] resulting in strains that respond poorly in pharmacodynamic models [50, 51]. Related FKS1 mutations have been found in other Candida species. Only in C. glabrata, conserved hot-spot mutations are found in both FKS1 (Phe625, Ser629) and FKS2 (Phe659, Ser663) with the latter occurring with twice the frequency [48].

Azoles inhibit lanosterol $14 \alpha$-demethylase, which is encoded by ERG11 (CYP51A in Aspergillus). Triazole antifungal agents differ in affinities for their drug target, which in turn influences their spectrum of activity. Fluconazole shows the weakest interaction and displays the narrowest spectrum, as it is active against yeasts but not molds. As such, it promotes the broadest resistance. In a recent study of 63 fluconazole-resistant clinical isolates, 55 isolates carried at least one mutation in ERG11, representing 26 distinct amino acid substitutions [52]. In contrast, highly active triazoles (e.g., voriconazole, posaconazole) interact more strongly with the drug target, show broader activity against yeasts and molds, and reveal a narrower spectrum of resistance mutations. To date, more than 70 amino acid substitutions have been described in Erg11 (or Cyp51A) from azole-resistant clinical isolates of C. albicans [53-58], A. fumigatus [24, 59, 60], and Cryptococcus neoformans [61, 62]. Within the azole family, chemical diversity around a core unit facilitates differential susceptibility and also resistance cross-reactivity. For example, some mutations in ERG11 result in fluconazole resistance only, others confer resistance to voriconazole but not posaconazole, and some display pan-resistance. Computational modeling using high-resolution structures as a template helps explain the impact of specific amino acid substitutions on drug-target interactions [63]. Recently, such modeling studies were greatly enhanced by the elucidation of a high-resolution Erg11 structure from baker's yeast [64••]. Drugs like posaconazole fill the structural space occupied by the substrate lanosterol, where they make a coordination bond with the heme iron extending from the active site to beyond the mouth of the entry channel (Fig. 3). A majority of mutations cluster in three main regions [65] with most substitutions altering the juxtaposition of drug with the heme cofactor. The structure of the active site and substrate channel helps account for the susceptibility observed for some prominent resistant mutants $[64 \cdot \bullet, 66]$.

Finally, in Aspergillus, mutations in Cyp51A are sufficient to induce resistance to some or all highly active triazole drugs, while in C. glabrata, target site mutations in CgERG11 do not 


\section{Azole-sensitive population}

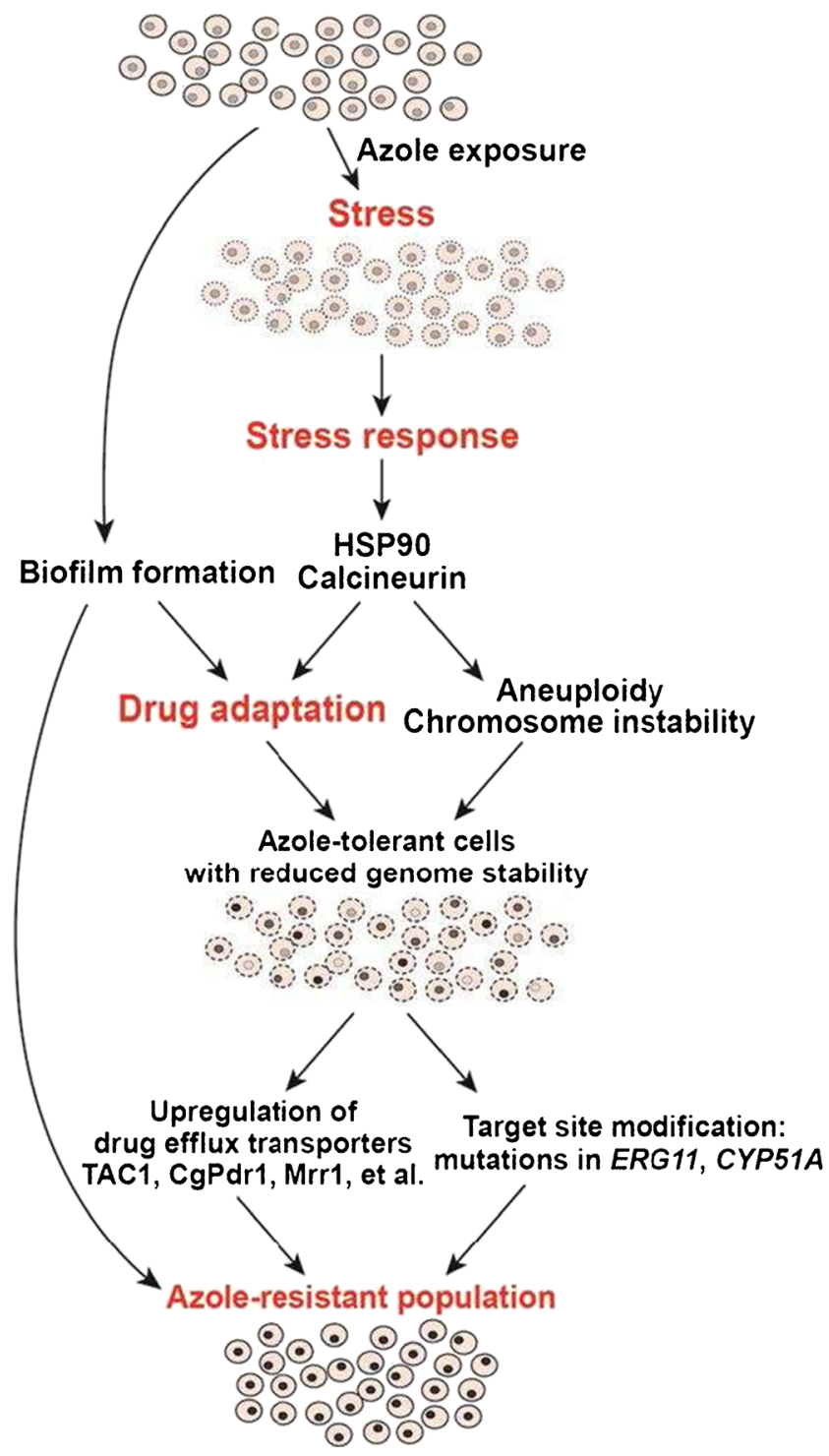

Fig. 2 Exposure to azole drugs triggers fungal stress responses that promote fungal adaptation and drug tolerance and, ultimately, emergence of stable genetic alterations that confer drug resistance. The HSP90 protein chaperone and its client, protein phosphatase calcineurin, are key stress signal transduction molecules that both upregulate pathways leading to drug tolerance and promote genome instability, increasing the likelihood of generating drug-resistant strains. Fungal biofilms, which readily form in vivo, are intrinsically resistant to azoles due to drug sequestration within the extracellular matrix and expression of drug efflux transporters

contribute to clinical resistance. In some organisms, mutations in Erg11 are but a first step toward higher-level resistance involving other resistance mechanisms such as target upregulation or overexpression of drug pumps.

\section{Decreased Intracellular Drug Levels}

As drugs need to reach their cellular targets to be effective, certain antifungals use permeases for cell entry including 5- fluorocytosine, which uses $F C A 1, F C Y 2, F C Y 22$, and $F C Y 23$ to enter $C$. albicans cells [50]. Fluconazole is believed to enter cells by an uncharacterized energy-independent facilitated diffusion mechanisms [67]. Modification of these uptake systems would confer drug resistance. Among the most common mechanisms for reducing cellular drug levels, energydependent drug efflux transporters recognize and extrude diverse chemical classes. Two different drug efflux systems modulate azole resistance, the ATP-binding cassette (ABC) superfamily and the major facilitator superfamily (MFS). The ATP-dependent transporters (ABC) are comprised of two transmembrane and two cytoplasmic nucleotide-binding domains, which catalyze ATP hydrolysis. Fungal genomes encode numerous $\mathrm{ABC}$ transporters, as they are presumed to purge the cell of toxic compounds and metabolites. C. albicans is predicted to contain $28 \mathrm{ABC}$ proteins [68], C. glabrata has 18, and A. fumigatus and C. neoformans have many more [69]. Despite their prevalence, only a few contribute to antifungal resistance. The PDR class comprises the major transporters involved in azole resistance including C. albicans CDR1 and CDR2 [70]; $\mathrm{CgCdr1}, \mathrm{CgCdr} 2$, and CgSnq2 in C. glabrata; and Afr1 in C. neoformans [71]. In $A$. fumigatus, $\mathrm{ABC}$ transporter genes are upregulated in response to azole exposure (AfuMDR1 (CDR1B), AfuMDR2, $a b c A-E)[72,73]$ and in resistant clinical isolates [74-76]. MFS transporters have multiple (12 or 14) transmembrane domains and use proton-motive force to drive drug efflux. The $C$. albicans genome predicts 95 MFS transporters in 17 families [77] but only one transporter gene, MDR1, is associated azole resistance [78-80]. In A. fumigatus, AfuMDR3 is upregulated in some itraconazole-resistant mutants [75]. It is unclear in Aspergillus whether induction of an ABC or MFS transporter is sufficient for resistance.

\section{Regulation of Drug Transporters}

Transcriptional regulation of $\mathrm{ABC}$ and MFS multidrug transporters is complex, involving cis- and trans-regulatory elements. Cis-acting elements regulate $C D R 1, C D R 2$, and $M D R 1$ in C. albicans, with the promoters of $C D R 1$ and $C D R 2$ containing common Drug Responsive Element (DRE) sequences that are required for transcriptional upregulation [81]. MDR1 cis-acting elements have complex arrangements that differ depending on the inducer [82]. In C. glabrata, pleiotropic DREs are present in $C g C D R 1$, $C g C D R 2$, and $C g S N Q 2$ [83, 84], and help confer high level CDR1 expression [85]. The first major trans-acting transcription element regulating efflux is $C$. albicans Transcriptional Activator of CDR (TAC1), a member of the $\mathrm{Zn}_{2} \mathrm{Cys}_{6}$ transcription factor family. Gain-of-function (GOF) mutations in $T A C 1$ [86, 87$]$ are responsible for the upregulation of $C D R 1$ and $C D R 2$ in azole-resistant isolates [88-90]. Tac1 binds to the DRE of $C D R 1$ and $C D R 2$, likely via a consensus-binding 
Table 1 Overview of Fks hot spot sequences and amino acid sequence positions resulting in echinocandin resistance

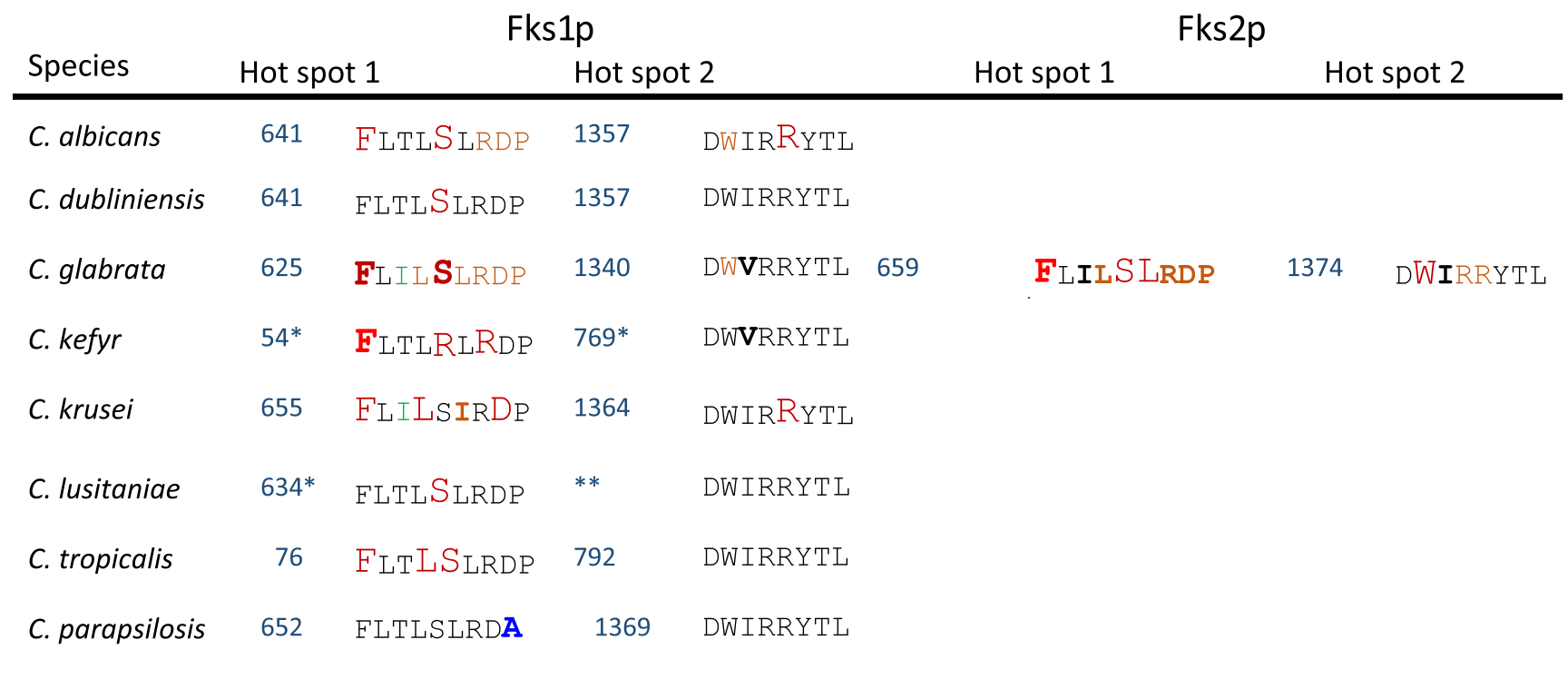

First amino acid number is shown for each hot-spot sequence

Amino acids in bold large red letters signify most prominent resistance

Amino acids in red-brown indicate weaker resistance

Amino acid in blue is a naturally occurring polymorphism with weak resistance

Amino acids in bold indicate strong resistance

Amino acids in green indicate silent mutation, acquired or naturally occurring

Amino acids in brown indicate naturally occurring mutation of unknown impact

*Indicates amino acid position based on partial sequence, sequencing of entire gene is required

**denotes separated sequences of HS1 and HS2, thus annotation of HS2 is nonsense

motif. Similarly, another $C D R 1$ regulator $M R R 2$ is required for the basal expression of CDR1 [91]. In C. glabrata, drug pump overexpression is the major mechanism responsible for azole resistance, and transcriptional activator $\mathrm{CgPdr} 1 \mathrm{reg}-$ ulates expression of $\mathrm{CgCDR} 1$ and $\mathrm{Cg} C D R 2$ [92]. CgPdr1 binds to the PDRE consensus in $C g C D R 1$ [85] and GOF mutations hyper-activate $\mathrm{CgPdr} 1$ upregulating $\mathrm{ABC}$ transporters [92-96]. Nearly 60 GOF mutations have been identified in $C g P D R 1$ alleles from clinical azole-resistant isolates [94]. In C. albicans, the $\mathrm{Zn}_{2} \mathrm{Cys}_{6}$ transcription factor Multidrug Resistance Regulator 1 (Mrr1) regulates MFS transporter gene MDR1 [97] and at least 15 different MRR1 GOF mutations are known [88, 98] to cause constitutive upregulation of MDRI [99]. Other positive regulators of MDR in C. albicans include Cap1 [100, 101] and Mcm1 [102, 103].



Fig. 3 Binding of lanosterol and itraconazole within active site heme region Erg11 from $S$. cerevisiae. a Lanosterol binding and coordination with heme shown with electron density profile. b Itraconazole binding to same region shown with electron density. c Bound itraconazole and amino acids commonly mutations to confer resistance. Adapted from Monk et al. [64••] 


\section{Chromosomal Anomalies}

It is now recognized that azole resistance in C. albicans and other Candida species is associated with a variety of largescale genomic alterations, including loss of heterozygosity $(\mathrm{LOH})$ involving specific genomic regions, increased chromosomal copy number, and aneuploidies. $\mathrm{LOH}$ is associated with resistance factors $E R G 11, T A C 1$, and $M R R 1$. It has been shown that mutations in these genes arise in a heterozygous state and are converted to homozygous form by LOH [104, 105]. Isochromosome formation is a separate and more pronounced genomic change. It increases gene copy numbers, and hence gene expression of azole resistance genes and Erg11, the azole target. Isochromosome formation on the left arm of chromosome $5 \mathrm{i}(5 \mathrm{~L})$ increases the copy number of ERG11 and TAC1 [106]. Similarly, the isochromosome variant $3 \mathrm{i}(3 \mathrm{R})$ on the right arm of chromosome 3 contains $C D R 1$ and $M R R 1$ [107]. An examination of 57 clinical C. albicans strains, disomic or monosomic for $\mathrm{Ch} 5$, found that the monosomy of Ch5 caused elevated levels of cell wall chitin and repressed levels of 1,3-beta-glucan, as well as diminished membrane ergosterol. This resulted in decreased susceptibility to caspofungin and increased susceptibility to fluconazole and amphotericin B [108]. Chromosomal alterations resulting in resistance are also observed with C. glabrata [109]. In C. neoformans, azole resistance is associated with disomies of chromosomes 1 and 4, which contain ERG11 and ABC transporter AFR1 [110]. Hetero-resistance, observed in C. albicans [111], relates to subpopulations within the same clone that vary in resistance based on the frequent loss and gain of chromosomes in response to selection in C. neoformans [112]. C. neoformans is heteroresistant to azoles due to transient duplications of whole chromosomes that carry the genes for azole resistance [110]. Chromosome 1, which harbors ERG11 and $A F R 1$, encoding the azole target and an $\mathrm{ABC}$ transporter, respectively, is the first one to be duplicated resulting in elevated MICs; further increases in MIC result from the duplication of Chr4.

\section{Stress Responses and Drug Adaptation}

Fungi are remarkably adaptive and have numerous genetic mechanisms that help protect against cellular stresses, such as those encountered following exposure to an antifungal agent. These stress adaptation responses frequently result in elevated in vitro MICs. Typically, the increased MIC is insufficient to confer clinical resistance resulting in breakthrough infections. Rather, stress adaptation stabilizes the cell in the presence of drug and allows it to develop more profound resistance mechanisms over time that are manifested as clinical resistance (Fig. 2). As first described for azoles, Hsp90 and calcineurin are two key cellular regulators critical for orchestrating cellular responses to drug-induced stress [113, 114•]. Hsp90 is a molecular chaperone that regulates the stability and function of diverse client proteins and controls stress responses by stabilizing the protein phosphatase calcineurin [115]. Calcineurin-Crz1 signaling influences a wide range of cellular response functions including ion homeostasis and cell wall biogenesis [116]. Compromising the function of Hsp90 or calcineurin can induce fungistatic drugs to become fungicidal enhancing efficacy. Thus, inhibition of Hsp90 or calcineurin may present a strategy to enhance the efficacy of azoles against resistant fungi [117]. Hsp90 and calcineurin-Crz1 signaling also contribute to echinocandin resistance in Candida species $[118,119]$. The cell's response to echinocandin action is highly robust, as numerous cellular responses are linked to maintaining cell wall integrity including $P K C$, calcineurin/ Crz1, and $H O G$ [120, 121]. Other responses such as modulation of sphingolipid biosynthesis result in a mixed phenotype involving resistance to caspofungin and hypersensitivity to micafungin [122]. Echinocandin action also results in pronounced compensatory increases in chitin synthesis, to help sustain the cell wall. Mutants with increased chitin content are less susceptible to caspofungin [120, 121, 123] and increased chitin biosynthesis has been partly invoked to account for paradoxical growth at high drug levels [124] [125]. In recent years, whole genome sequencing of serial isolates has been used to determine genetic signatures related to evolution of resistance. Whole genome sequencing of $C$. glabrata isolates before and after caspofungin treatment and breakthrough identified expected FKS mutations and HSP90 effects. In addition, it identified mutations in genes MOH1, GPH1, CDC6, and $T C B 1 / 2 ; c d c 6$ mutations were independently shown to have a role in echinocandin susceptibility [119]. In total, these responses help confer drug adaption, which predispose cells for higher resistance such as the formation of a stable $F K S$ mutation.

\section{Genetic Plasticity as a Driver of Resistance}

C. albicans can develop azole resistance by acquiring chromosomal disomies or segmental chromosomal duplications involving the chromosomes carrying azole target ERG11 and drug efflux genes $[106,126]$. Acquisition of multiple chromosome disomies upon azole exposure was also observed in C. neoformans [110]. However, appearance of significant genomic alterations is not specific to azoles, but also occurs in the presence of other types of stress. The genetic changes underlying antifungal drug resistance do not arise in a random manner, as they are promoted by varying stress inducers including antifungal drugs and host immunity. For instance, in C. albicans, elevated temperature and oxidative stress 
promote aneuploidy and chromosome arm homozygosis [127]. In Saccharomyces cerevisiae, several different stresses, including oxidative, translational, and ER stress, promote chromosome loss and appearance of marked karyotype diversity [128]. Consistent with these observations, passage of $C$. albicans through the mouse promotes genome rearrangements in the fungus even in the absence of antifungal treatment, suggesting that this genetic instability is due to conditions encountered within the host [129]. Furthermore, analysis of C. glabrata clinical isolates indicates that this organism undergoes drastic genome rearrangements with multiple chromosomal translocations and appearance of new chromosomes [130]. Clinical isolates of C. glabrata have highly variable genomes [109, 126] suggesting that this species possesses mechanisms that specifically promote and/or help the cells tolerate extensive genetic changes in response to stress. Several studies suggest that an increase in the proportion of aneuploid cells happens early in response to stress [131, 132]. One factor involved in this process is HSP90, whose inhibition strongly reduces stress-induced aneuploidy and drug resistance in C. albicans and S. cerevisiae [115, 128]. Formation of aneuploidy is followed by smaller-scaled genetic changes, such as insertions, deletions, and point mutations in individual genes. What drives such changes is not well understood, although it has been shown that aneuploidy itself can promote other types of genetic alterations, possibly because it alters gene dosage of a subset of the genome, thus altering complexes involved in chromosome maintenance and DNA repair [133].

\section{Drivers of Resistance}

The development of antifungal resistance is a complex process involving the host, drug, and microbial factors, which collectively contribute to therapeutic failure. Host immune status is important as the immune system must work in concert with antifungal drugs to control an infection. Severe immune dysfunction results in patients less responsive to treatment since microbial burdens are larger and the drug must combat the infection without immune support. Surgical devices such as indwelling catheters and artificial heart valves provide surfaces for infecting fungi to establish biofilms that restrict drug access. The site of the infection contributes to clinical resistance, since it may be inaccessible to drugs. Successful therapy requires that the drug reach its microbial target with a suitable potency but this is often unknown. Blood levels of drugs may not accurately predict whether a drug reaches the primary site of infection, as it is difficult to deliver drugs at an adequate concentration to certain infected tissues and organs. Abdominal candidiasis is a high burden infection in which drug access is restricted, which leads to breakthrough infections [134]. In some cases, drugs that are highly serum protein bound, such as the echinocandins, have altered antifungal properties whereby in vitro fungicidal drugs can act as fungistatic agents in vivo [135]. In recent years, the role of environment as a driver for resistance has become prominent. As described earlier for $A$. fumigatus, triazole resistance due to two prominent modifications of Cyp51A, $\mathrm{TR}_{34} / \mathrm{L} 98 \mathrm{H}[10$, 136-139], and $\mathrm{TR}_{46} / \mathrm{Y} 121 \mathrm{~F} / \mathrm{T} 289 \mathrm{~A}$ [23] arose as a consequence of azole use in the agricultural world [11]. As Aspergillus spores emerge from the environment, this environmentally driven resistance is spreading throughout Europe, India, and Asia [19, 140, 141]. Finally, like all antiinfectives, patient compliance is critical for effective treatment, as poor adherence to drug regimens reduce drug effectiveness, contributing to resistance. Overall, there remains a strong relationship between drug exposure and the emergence of resistance. The development of echinocandin resistance in Candida species typically requires prolonged drug exposure [142-145]. But it can also arise rapidly after the start of therapy $[146,147]$. Horizontal transmission of resistant strains is not generally observed most likely because they carry a fitness cost. With FKS mutants, decreased glucan synthase activity results in less robust cells with modified cell walls [148, 49]. These FKS mutant strains are less virulent and compete poorly with their wild-type counterparts [48, 149, 148]. Lastly, as total drug exposure is a critical factor influencing resistance emergence, prophylaxis has emerged as a concern. Fluconazole and the echinocandins caspofungin and micafungin are excellent prophylaxis agents against invasive candidiasis because they have favorable pharmacokinetics and safety profile. However, the expanding use of antifungal prophylaxis increases patient exposure to drugs, and it is not surprising that it promotes the emergence of resistance in certain clinical settings.

\section{Conclusion}

Overall, antifungal drug resistance due to acquired mechanisms is an uncommon event, as most infecting species retain drug susceptibility. However, acquired drug resistance can be a critical factor in some settings with critically ill patients, and the emergence of significant multidrug resistance involving azoles and echinocandins in organisms such as C. glabrata is troubling. The mechanisms conferring drug resistance are now well defined, and ongoing studies are seeking to identify genetic factors that can influence their emergence. Fungi have evolved to respond to stress in a highly dynamic manner, ranging from specific point mutations to major chromosomal modifications that directly and indirectly influence induction of specific resistance mechanisms. There is now a strong appreciation that stress responses promote drug adaptation, which by itself does not lead to clinical failure but can 
ultimately lead to development of higher-level resistance and diminished clinical response (Fig. 2). Finally, in recent years, anatomical reservoirs that restrict drug access or promote biofilm formation have been identified to be important contributors to resistance emergence in the clinic. As new molecular tools have emerged, there is now an opportunity to detect drug resistance earlier and develop therapeutic strategies to avoid or mitigate resistance.

\section{Compliance with Ethics Guidelines}

Conflict of Interest Dr. Perlin reports grants and personal fees from Astellas Pharmaceutical, grants from CIDARA, grants from Scynexis, outside the submitted work; in addition, Dr. Perlin has a patent Assays for Resistance to Echinocandin-Class Drugs issued, a patent ASSAYS FOR FUNGAL INFECTION pending, a patent NEAR INFRARED LABELS AND METHODS OF USE THEREOF pending, and a patent METHODS TO DETECT A FUNGAL CELL pending.

Dr. Shor and Dr. Zhao both declare they have no conflicts of interest to disclose.

Human and Animal Rights and Informed Consent This article contains no studies with human or animal subjects performed by the author.

\section{References}

Papers of particular interest, published recently, have been highlighted as:

- Of importance

-• Of major importance

1. Brown GD et al. Hidden killers: human fungal infections. Sci Transl Med. 2012;4(165):165rv13.

2. Pfaller MA, Diekema DJ. Rare and emerging opportunistic fungal pathogens: concern for resistance beyond Candida albicans and Aspergillus fumigatus. J Clin Microbiol. 2004;42(10):4419-31.

3. Diekema $\mathrm{D}$ et al. The changing epidemiology of healthcareassociated candidemia over three decades. Diagn Microbiol Infect Dis. 2012;73(1):45-8.

4. Pfaller MA et al. Bloodstream infections due to Candida species: SENTRY antimicrobial surveillance program in North America and Latin America, 1997-1998. Antimicrob Agents Chemother. 2000;44(3):747-51.

5. Hope W, Morton A, Eisen DP. Increase in prevalence of nosocomial non-Candida albicans candidaemia and the association of Candida krusei with fluconazole use. J Hosp Infect. 2002;50(1): 56-65.

6. Pfaller MA et al. Candida guilliermondii, an opportunistic fungal pathogen with decreased susceptibility to fluconazole: geographic and temporal trends from the ARTEMIS DISK antifungal surveillance program. J Clin Microbiol. 2006;44(10):3551-6.

7. Pavie J et al. Breakthrough disseminated Aspergillus ustus infection in allogeneic hematopoietic stem cell transplant recipients receiving voriconazole or caspofungin prophylaxis. J Clin Microbiol. 2005;43(9):4902-4.

8. Balajee SA et al. Aspergillus fumigatus variant with decreased susceptibility to multiple antifungals. Antimicrob Agents Chemother. 2004;48(4):1197-203.
9. Balajee SA et al. Aspergillus lentulus sp. nov., a new sibling species of A. fumigatus. Eukaryot Cell. 2005;4(3):625-32.

10. Snelders E et al. Emergence of azole resistance in Aspergillus fumigatus and spread of a single resistance mechanism. PLoS Med. 2008;5(11):e219.

11. Verweij PE et al. Azole resistance in Aspergillus fumigatus: a sideeffect of environmental fungicide use? Lancet Infect Dis. 2009;9(12):789-95.

12. Camps SM et al. Molecular epidemiology of Aspergillus fumigatus isolates harboring the TR34/L98H azole resistance mechanism. J Clin Microbiol. 2012;50(8):2674-80.

13. Denning DW, Perlin DS. Azole resistance in Aspergillus: a growing public health menace. Future Microbiol. 2011;6(11):1229-32.

14. Arendrup MC, Perlin DS. Echinocandin resistance: an emerging clinical problem? Curr Opin Infect Dis. 2014;27(6):484-92.

15. Vermeulen E, Lagrou K, Verweij PE. Azole resistance in Aspergillus fumigatus: a growing public health concern. Curr Opin Infect Dis. 2013;26(6):493-500.

16. Denning DW et al. Itraconazole resistance in Aspergillus fumigatus. Antimicrob Agents Chemother. 1997;41(6):1364-8.

17. Snelders E, Melchers WJ, Verweij PE. Azole resistance in Aspergillus fumigatus: a new challenge in the management of invasive aspergillosis? Future Microbiol. 2011;6(3):335-47.

18. Morio F., et al., High prevalence of triazole resistance in Aspergillus fumigatus, especially mediated by TR/L98H, in a French cohort of patients with cystic fibrosis. J Antimicrob Chemother, 2012. 67(8): 1870-3

19. Bader $\mathrm{O}$ et al. cyp51A-based mechanisms of Aspergillus fumigatus azole drug resistance present in clinical samples from Germany. Antimicrob Agents Chemother. 2013;57(8):3513-7.

20. Chowdhary A et al. Isolation of multiple-triazole-resistant Aspergillus fumigatus strains carrying the TR/L98H mutations in the cyp51A gene in India. J Antimicrob Chemother. 2012;67(2): $362-6$.

21. Seyedmousavi $\mathrm{S}$ et al. Azole-resistant Aspergillus fumigatus, Iran. Emerg Infect Dis. 2013;19(5):832-4.

22. Vermeulen E et al., Azole-resistant Aspergillus fumigatus due to TR46/Y121F/T289A mutation emerging in Belgium, July 2012. Euro Surveill, 2012. 17(48).

23. van der Linden JW et al. Aspergillosis due to voriconazole highly resistant Aspergillus fumigatus and recovery of genetically related resistant isolates from domiciles. Clin Infect Dis. 2013;57(4): 513-20.

24. Howard SJ et al. Frequency and evolution of azole resistance in Aspergillus fumigatus associated with treatment failure. Emerg Infect Dis. 2009;15(7):1068-76.

25. Bueid A et al. Azole antifungal resistance in Aspergillus fumigatus: 2008 and 2009. J Antimicrob Chemother. 2010;65(10):2116-8.

26. Pfaller MA et al. Echinocandin and triazole antifungal susceptibility profiles for Candida spp., Cryptococcus neoformans, and Aspergillus fumigatus: application of new CLSI clinical breakpoints and epidemiologic cutoff values to characterize resistance in the SENTRY Antimicrobial Surveillance Program (2009). Diagn Microbiol Infect Dis. 2011;69(1):45-50.

27. Pfaller MA et al. Candida bloodstream infections: comparison of species distribution and resistance to echinocandin and azole antifungal agents in intensive care unit (ICU) and non-ICU settings in the SENTRY Antimicrobial Surveillance Program (20082009). Int J Antimicrob Agents. 2011;38(1):65-9.

28. Pfaller MA et al. Geographic variations in species distribution and echinocandin and azole antifungal resistance rates among Candida bloodstream infection isolates: report from the SENT RY Antimicrobial Surveillance Program (2008 to 2009). J Clin Microbiol. 2011;49(1):396-9. 
29. Castanheira $\mathrm{M}$ et al. Low prevalence of fks1 hot spot 1 mutations in a worldwide collection of Candida strains. Antimicrob Agents Chemother. 2010;54(6):2655-9.

$30 .-$ Alexander BD et al. Increasing echinocandin resistance in Candida glabrata: clinical failure correlates with presence of FKS mutations and elevated minimum inhibitory concentrations. Clin Infect Dis. 2013;56(12):1724-32

This clinical study demonstrates the prominent increase in echinocandin resistance among Candida glabrata isolates with a parallel increase in coresistance to azole antifungal drugs.

31. Pfaller MA et al. Frequency of decreased susceptibility and resistance to echinocandins among fluconazole-resistant bloodstream isolates of Candida glabrata. J Clin Microbiol. 2012;50(4): 1199-203.

32. Pham CD et al. Role of FKS mutations in Candida glabrata: MIC values, echinocandin resistance, and multidrug resistance. Antimicrob Agents Chemother. 2014;58(8):4690-6.

33. Eschenauer GA et al. Real-world experience with echinocandin MICs against Candida species in a multicenter study of hospitals that routinely perform susceptibility testing of bloodstream isolates. Antimicrob Agents Chemother. 2014;58(4):1897-906.

34. Kumamoto CA. Candida biofilms. Curr Opin Microbiol. 2002;5(6):608-11.

35. Ramage $\mathrm{G}$ et al. Our current understanding of fungal biofilms. Crit Rev Microbiol. 2009;35(4):340-55.

36. Nett JE et al. Role of Fks1p and matrix glucan in Candida albicans biofilm resistance to an echinocandin, pyrimidine, and polyene. Antimicrob Agents Chemother. 2010;54(8):3505-8.

37. Chandra J, Zhou G, Ghannoum MA. Fungal biofilms and antimycotics. Curr Drug Targets. 2005;6(8):887-94.

38. Fanning S, Mitchell AP. Fungal biofilms. PLoS Pathog. 2012;8(4):e1002585.

39. Mukherjee PK et al. Mechanism of fluconazole resistance in Candida albicans biofilms: phase-specific role of efflux pumps and membrane sterols. Infect Immun. 2003;71(8):4333-40.

40. Silva $\mathrm{S}$ et al. Biofilms of non-Candida albicans Candida species: quantification, structure and matrix composition. Med Mycol. 2009;47(7):681-9.

41. Nett JE et al. Genetic basis of Candida biofilm resistance due to drug-sequestering matrix glucan. J Infect Dis. 2010;202(1):171-5.

42. Nett J.E., et al., Interface of Candida albicans biofilm matrixassociated drug resistance and cell wall integrity regulation. Eukaryot Cell, 2011. 10(12): 1660-9

43. Desai JV et al. Regulatory role of glycerol in Candida albicans biofilm formation. MBio. 2013;4(2):e00637-12.

44. Nobile CJ et al. Biofilm matrix regulation by Candida albicans Zap1. PLoS Biol. 2009;7(6):e1000133.

45. Niimi K et al. Overexpression of Candida albicans CDR1, CDR2, or MDR1 does not produce significant changes in echinocandin susceptibility. Antimicrob Agents Chemother. 2006;50(4): 1148-55.

46. Perlin DS. Current perspectives on echinocandin class drugs. Future Microbiol. 2011;6(4):441-57.

47. Park $\mathrm{S}$ et al. Specific substitutions in the echinocandin target Fks1p account for reduced susceptibility of rare laboratory and clinical Candida sp. isolates. Antimicrob Agents Chemother. 2005;49(8):3264-73

This work provides the foundation for the role of FKS mutations as the principal mechanism associated with clinical echincaondin resistance.

48. Garcia-Effron $\mathrm{G}$ et al. Effect of Candida glabrata FKS1 and FKS2 mutations on echinocandin sensitivity and kinetics of 1 , 3-beta-D-glucan synthase: implication for the existing susceptibility breakpoint. Antimicrob Agents Chemother. 2009;53(9): 3690-9.
49. Garcia-Effron G, Park S, Perlin DS. Correlating echinocandin MIC and kinetic inhibition of fks1 mutant glucan synthases for Candida albicans: implications for interpretive breakpoints. Antimicrob Agents Chemother. 2009;53(1):112-22.

50. Hope WW et al. Molecular mechanisms of primary resistance to flucytosine in Candida albicans. Antimicrob Agents Chemother. 2004;48(11):4377-86.

51. Wiederhold NP et al. Caspofungin dose escalation for invasive candidiasis due to resistant Candida albicans. Antimicrob Agents Chemother. 2011;55(7):3254-60.

52. Flowers SA et al. Contribution of clinically derived mutations in ERG11 to azole resistance in Candida albicans. Antimicrob Agents Chemother. 2015;59(1):450-60.

53. Perea $\mathrm{S}$ et al. Prevalence of molecular mechanisms of resistance to azole antifungal agents in Candida albicans strains displaying high-level fluconazole resistance isolated from human immunodeficiency virus-infected patients. Antimicrob Agents Chemother. 2001;45(10):2676-84.

54. White TC. The presence of an R467K amino acid substitution and loss of allelic variation correlate with an azole-resistant lanosterol 14alpha demethylase in Candida albicans. Antimicrob Agents Chemother. 1997;41(7):1488-94.

55. Xu Y, Chen L, Li C. Susceptibility of clinical isolates of Candida species to fluconazole and detection of Candida albicans ERG11 mutations. J Antimicrob Chemother. 2008;61(4):798-804.

56. Kelly SL, Lamb DC, Kelly DE. Y132H substitution in Candida albicans sterol 14alpha-demethylase confers fluconazole resistance by preventing binding to haem. FEMS Microbiol Lett. 1999;180(2):171-5.

57. Kelly SL et al. The G464S amino acid substitution in Candida albicans sterol 14alpha-demethylase causes fluconazole resistance in the clinic through reduced affinity. Biochem Biophys Res Commun. 1999;262(1):174-9.

58. Asai $\mathrm{K}$ et al. Formation of azole-resistant Candida albicans by mutation of sterol 14-demethylase P450. Antimicrob Agents Chemother. 1999;43(5):1163-9.

59. Howard SJ, Arendrup MC. Acquired antifungal drug resistance in Aspergillus fumigatus: epidemiology and detection. Med Mycol. 2011;49 Suppl 1:S90-5.

60. Verweij PE et al. Azole-resistance in Aspergillus: proposed nomenclature and breakpoints. Drug Resist Updat. 2009;12(6): 141-7.

61. Rodero L et al. G484S amino acid substitution in lanosterol 14alpha demethylase (ERG11) is related to fluconazole resistance in a recurrent Cryptococcus neoformans clinical isolate. Antimicrob Agents Chemother. 2003;47(11):3653-6.

62. Sionov E et al. Identification of a Cryptococcus neoformans cytochrome P450 lanosterol 14alpha-demethylase (Erg11) residue critical for differential susceptibility between fluconazole/voriconazole and itraconazole/posaconazole. Antimicrob Agents Chemother. 2012;56(3):1162-9.

63. Xiao L et al. Three-dimensional models of wild-type and mutated forms of cytochrome P450 14alpha-sterol demethylases from Aspergillus fumigatus and Candida albicans provide insights into posaconazole binding. Antimicrob Agents Chemother. 2004;48(2):568-74.

64.• Monk BC et al. Architecture of a single membrane spanning cytochrome P450 suggests constraints that orient the catalytic domain relative to a bilayer. Proc Natl Acad Sci U S A. 2014; 111(10):3865-70

The work describes for the first time a high resolution structure of the 14 alpha-demethylase, which is the target for azole antifungal drugs.

65. Favre B, Didmon M, Ryder NS. Multiple amino acid substitutions in lanosterol 14alpha-demethylase contribute to azole resistance in Candida albicans. Microbiology. 1999;145(Pt 10):2715-25. 
66. Snelders E et al. Azole resistance profile of amino acid changes in Aspergillus fumigatus CYP51A based on protein homology modeling. Antimicrob Agents Chemother. 2010;54(6): 2425-30.

67. Mansfield B. et al., Azole drugs are imported by facilitated diffusion in Candida albicans and other pathogenic fungi. PLoS Pathog, 2010. 6(9): e1001126.

68. Prasad R, Goffeau A. Yeast ATP-binding cassette transporters conferring multidrug resistance. Annu Rev Microbiol. 2012;66(1): 39-63.

69. Lamping E et al. Fungal PDR transporters: phylogeny, topology, motifs and function. Fungal Genet Biol. 2010;47(2):127-42.

70. Braun BR et al. A human-curated annotation of the Candida albicans genome. PLoS Genet. 2005;1(1):36-57.

71. Coleman JJ, Mylonakis E. Efflux in Fungi: La Pièce de Résistance. PLoS Pathog. 2009;5(6):e1000486.

72. Tobin MB, Peery RB, Skatrud PL. Genes encoding multiple drug resistance-like proteins in Aspergillus fumigatus and Aspergillus flavus. Gene. 1997;200(1-2):11-23.

73. da Silva Ferreira ME et al. Transcriptome analysis of Aspergillus fumigatus exposed to voriconazole. Curr Genet. 2006;50(1): 32-44.

74. Slaven JW et al. Increased expression of a novel Aspergillus fumigatus $\mathrm{ABC}$ transporter gene, atrF, in the presence of itraconazole in an itraconazole resistant clinical isolate. Fungal Genet Biol. 2002;36(3):199-206.

75. Nascimento AM et al. Multiple resistance mechanisms among Aspergillus fumigatus mutants with high-level resistance to itraconazole. Antimicrob Agents Chemother. 2003;47(5): 1719-26.

76. Fraczek MG et al. The cdr1B efflux transporter is associated with non-cyp51a-mediated itraconazole resistance in Aspergillus fumigatus. J Antimicrob Chemother. 2013;68(7):1486-96.

77. Gaur M et al. MFS transportome of the human pathogenic yeast Candida albicans. BMC Genomics. 2008;9:579.

78. Lamping $\mathrm{E}$ et al. Characterization of three classes of membrane proteins involved in fungal azole resistance by functional hyperexpression in Saccharomyces cerevisiae. Eukaryot Cell. 2007;6(7):1150-65.

79. Sanglard D et al. Susceptibilities of Candida albicans multidrug transporter mutants to various antifungal agents and other metabolic inhibitors. Antimicrob Agents Chemother. 1996;40(10): 2300-5.

80. Sanglard D et al. Mechanisms of resistance to azole antifungal agents in Candida albicans isolates from AIDS patients involve specific multidrug transporters. Antimicrob Agents Chemother. 1995;39(11):2378-86.

81. de Micheli $\mathrm{M}$ et al. A common drug-responsive element mediates the upregulation of the Candida albicans ABC transporters CDR1 and CDR2, two genes involved in antifungal drug resistance. Mol Microbiol. 2002;43(5):1197-214.

82. Rognon B et al. Identification of promoter elements responsible for the regulation of MDR1 from Candida albicans, a major facilitator transporter involved in azole resistance. Microbiology. 2006;152(Pt 12):3701-22.

83. Torelli $\mathrm{R}$ et al. The ATP-binding cassette transporter-encoding gene CgSNQ2 is contributing to the CgPDR1-dependent azole resistance of Candida glabrata. Mol Microbiol. 2008;68(1): 186-201.

84. Sanglard D et al. The ATP binding cassette transporter gene CgCDR1 from Candida glabrata is involved in the resistance of clinical isolates to azole antifungal agents. Antimicrob Agents Chemother. 1999;43(11):2753-65.

85. Paul S, Schmidt JA, Moye-Rowley WS. Regulation of the CgPdr1 transcription factor from the pathogen Candida glabrata. Eukaryot Cell. 2011;10(2):187-97.
86. Coste AT et al. TAC1, transcriptional activator of CDR genes, is a new transcription factor involved in the regulation of Candida albicans $\mathrm{ABC}$ transporters CDR1 and CDR2. Eukaryot Cell. 2004;3(6):1639-52

This work describes the underlying genetic mechanism responsible for the upregulation of ABC transporters CDR1 and CDR2, which confer azole resistance in Candida albicans.

87. Coste A et al. A mutation in Taclp, a transcription factor regulating CDR1 and CDR2, is coupled with loss of heterozygosity at chromosome 5 to mediate antifungal resistance in Candida albicans. Genetics. 2006;172(4):2139-56.

88. Sanglard D. Diagnosis of antifungal drug resistance mechanisms in fungal pathogens: transcriptional gene regulation. Curr Fungal Infect Rep. 2011;5:157-67.

89. Siikala E et al. Persistent Candida albicans colonization and molecular mechanisms of azole resistance in autoimmune polyendocrinopathy-candidiasis-ectodermal dystrophy (APECED) patients. J Antimicrob Chemother. 2010;65(12):2505-13.

90. Coste AT et al. Functional analysis of cis- and trans-acting elements of the Candida albicans CDR2 promoter with a novel promoter reporter system. Eukaryot Cell. 2009;8(8):1250-67.

91. Schillig R, Morschhauser J. Analysis of a fungus-specific transcription factor family, the Candida albicans zinc cluster proteins, by artificial activation. Mol Microbiol. 2013;89(5):1003-17.

92. Vermitsky J-P, Edlind TD. Azole resistance in Candida glabrata: coordinate upregulation of multidrug transporters and evidence for a Pdr1-like transcription factor. Antimicrob Agents Chemother. 2004;48(10):3773-81.

93. Caudle $\mathrm{KE}$ et al. Genomewide expression profile analysis of the Candida glabrata Pdr1 regulon. Eukaryot Cell. 2011;10(3):373-83.

94. Ferrari $\mathrm{S}$ et al. Gain of function mutations in CgPDR1 of Candida glabrata not only mediate antifungal resistance but also enhance virulence. PLoS Pathog. 2009;5(1):e1000268.

95. Tsai $\mathrm{H}$ et al. Candida glabrata PDR1, a transcriptional regulator of a pleiotropic drug resistance network, mediates azole resistance in clinical isolates and petite mutants. Antimicrob Agents Chemother. 2006;50(4):1384-92.

96. Vermitsky J-P et al. Pdr1 regulates multidrug resistance in Candida glabrata: gene disruption and genome-wide expression studies. Mol Microbiol. 2006;61(3):704-22.

97. Morschhauser J et al. The transcription factor Mrrlp controls expression of the MDR1 efflux pump and mediates multidrug resistance in Candida albicans. PLoS Pathog. 2007:3(11):e164.

98. Eddouzi J et al. Molecular mechanisms of drug resistance in clinical Candida species isolated from Tunisian hospitals. Antimicrob Agents Chemother. 2013;57(7):3182-93.

99. Dunkel $\mathrm{N}$ et al. Mutations in the multidrug resistance regulator MRR1, followed by loss of heterozygosity, are the main cause of MDR1 overexpression in fluconazole-resistant Candida albicans strains. Mol Microbiol. 2008;69(4):827-40.

100. Znaidi $\mathrm{S}$ et al. Identification of the Candida albicans Cap1p regulon. Eukaryot Cell. 2009;8(6):806-20.

101. Alarco AM, Raymond M. The bZip transcription factor Caplp is involved in multidrug resistance and oxidative stress response in Candida albicans. J Bacteriol. 1999;181(3):700-8.

102. Lavoie $\mathrm{H}$ et al. A toolbox for epitope-tagging and genomewide location analysis in Candida albicans. BMC Genomics. 2008;9:578.

103. Riggle P, Kumamoto C. Transcriptional regulation of MDR1, encoding a drug efflux determinant, in fluconazole-resistant Candida albicans strains through an $\mathrm{Mcm} 1 \mathrm{p}$ binding site. Eukaryot Cell. 2006;5(12):1957-68.

104. Selmecki A, Forche A, Berman J. Genomic plasticity of the human fungal pathogen Candida albicans. Eukaryot Cell. 2010;9(7): 991-1008. 
105. Coste A et al. Genotypic evolution of azole resistance mechanisms in sequential Candida albicans isolates. Eukaryot Cell. 2007;6(10): 1889-904.

106. Selmecki A, Forche A, Berman J. Aneuploidy and isochromosome formation in drug-resistant Candida albicans. Science. 2006;313(5785):367-70.

107. Selmecki AM et al. Acquisition of aneuploidy provides increased fitness during the evolution of antifungal drug resistance. PLoS Genet. 2009;5(10):e1000705.

108. Yang $\mathrm{F}$ et al. Chromosome 5 monosomy of Candida albicans controls susceptibility to various toxic agents, including major antifungals. Antimicrob Agents Chemother. 2013;57(10): 5026-36.

109. Poláková $\mathrm{S}$ et al. Formation of new chromosomes as a virulence mechanism in yeast Candida glabrata. Proc Natl Acad Sci U S A. 2009;106(8):2688-93.

110. Sionov E et al. Cryptococcus neoformans overcomes stress of azole drugs by formation of disomy in specific multiple chromosomes. PLoS Pathog. 2010;6(4):e1000848.

111. Marr K et al. Inducible azole resistance associated with a heterogeneous phenotype in Candida albicans. Antimicrob Agents Chemother. 2001;45(1):52-9.

112. Sionov E, Chang YC, Kwon-Chung KJ. Azole heteroresistance in Cryptococcus neoformans: emergence of resistant clones with chromosomal disomy in the mouse brain during fluconazole treatment. Antimicrob Agents Chemother. 2013;57(10):5127-30.

113. Cowen LE. Hsp90 orchestrates stress response signaling governing fungal drug resistance. PLoS Pathog. 2009;5(8): e1000471.

114. Cowen LE, Steinbach WJ. Stress, drugs, and evolution: the role of cellular signaling in fungal drug resistance. Eukaryot Cell. 2008;7(5):747-64

This work describes the importance of HSP90 as a stabilizing factor that contributes to tolerance and the development of antifungal drug resistance.

115. Cowen LE, Lindquist S. Hsp90 potentiates the rapid evolution of new traits: drug resistance in diverse fungi. Science. 2005;309(5744): 2185-9.

116. Thewes S. Calcineurin-Crz1 signaling in lower eukaryotes. Eukaryot Cell. 2014;13(6):694-705.

117. Cowen LE et al. Harnessing Hsp90 function as a powerful, broadly effective therapeutic strategy for fungal infectious disease. Proc Natl Acad Sci U S A. 2009;106(8):2818-23.

118. Singh SD et al. Hsp90 governs echinocandin resistance in the pathogenic yeast Candida albicans via calcineurin. PLoS Pathog. 2009;5(7):e1000532.

119. Singh-Babak SD et al. Global analysis of the evolution and mechanism of echinocandin resistance in Candida glabrata. PLoS Pathog. 2012;8(5):e1002718.

120. Munro $\mathrm{CA}$ et al. The $\mathrm{PKC}, \mathrm{HOG}$ and $\mathrm{Ca}^{2+}$ signalling pathways co-ordinately regulate chitin synthesis in Candida albicans. Mol Microbiol. 2007;63(5):1399-413.

121. Walker LA et al. Stimulation of chitin synthesis rescues Candida albicans from echinocandins. PLoS Pathog. 2008;4(4):e1000040.

122. Healey KR et al. CRS-MIS in Candida glabrata: sphingolipids modulate echinocandin-Fks interaction. Mol Microbiol. 2012;86(2):303-13.

123. Gow NA et al. Immune recognition of Candida albicans betaglucan by dectin-1. J Infect Dis. 2007;196(10):1565-71.

124. Stevens DA, Espiritu M, Parmar R. Paradoxical effect of caspofungin: reduced activity against Candida albicans at high drug concentrations. Antimicrob Agents Chemother. 2004;48(9): $3407-11$.

125. Stevens DA et al. Escape of Candida from caspofungin inhibition at concentrations above the MIC (paradoxical effect) accomplished by increased cell wall chitin; evidence for beta1,6-glucan synthesis inhibition by caspofungin. Antimicrob Agents Chemother. 2006;50(9):3160-1.

126. Shin JH et al. Changes in karyotype and azole susceptibility of sequential bloodstream isolates from patients with Candida glabrata candidemia. J Clin Microbiol. 2007;45(8): 2385-91.

127. Forche A. et al., Stress alters rates and types of loss of heterozygosity in Candida albicans. MBio, 2011. 2(4): e00129-11

128. Chen $\mathrm{G}$ et al. Hsp90 stress potentiates rapid cellular adaptation through induction of aneuploidy. Nature. 2012;482(7384): 246-50.

129. Forche A et al. Evolution in Candida albicans populations during a single passage through a mouse host. Genetics. 2009;182(3): 799-811.

130. Ahmad KM et al. Small chromosomes among Danish Candida glabrata isolates originated through different mechanisms. Antonie Van Leeuwenhoek. 2013;104(1):111-22.

131. Harrison BD et al. A tetraploid intermediate precedes aneuploid formation in yeasts exposed to fluconazole. PLoS Biol. 2014;12(3):e1001815.

132. Yona $\mathrm{AH}$ et al. Chromosomal duplication is a transient evolutionary solution to stress. Proc Natl Acad Sci U S A. 2012;109(51): 21010-5.

133. Sheltzer JM et al. Aneuploidy drives genomic instability in yeast. Science. 2011;333(6045):1026-30.

134. Shields RK et al. Abdominal candidiasis is a hidden reservoir of echinocandin resistance. Antimicrob Agents Chemother. 2014;58(12):7601-5.

135. Kovacs $\mathrm{R}$ et al. Killing rates exerted by caspofungin in $50 \%$ serum and its correlation with in vivo efficacy in a neutropenic murine model against Candida krusei and Candida inconspicua. J Med Microbiol. 2014;63(Pt 2):186-94.

136. Mellado $\mathrm{E}$ et al. A new Aspergillus fumigatus resistance mechanism conferring in vitro cross-resistance to azole antifungals involves a combination of cyp51A alterations. Antimicrob Agents Chemother. 2007;51(6):1897-904.

137. Snelders E et al. Possible environmental origin of resistance of Aspergillus fumigatus to medical triazoles. Appl Environ Microbiol. 2009;75(12):4053-7.

138. Snelders E et al. The structure-function relationship of the Aspergillus fumigatus cyp51A L98H conversion by site-directed mutagenesis: the mechanism of $\mathrm{L} 98 \mathrm{H}$ azole resistance. Fungal Genet Biol. 2011;48(11):1062-70.

139. van der Linden JW et al. Clinical implications of azole resistance in Aspergillus fumigatus, The Netherlands, 2007-2009. Emerg Infect Dis. 2011;17(10):1846-54.

140. Chowdhary A et al. Clonal expansion and emergence of environmental multiple-triazole-resistant Aspergillus fumigatus strains carrying the TR(3)(4)/L98H mutations in the cyp51A gene in India. PLoS ONE. 2012;7(12):e52871.

141. Arikan-Akdagli S. Azole resistance in Aspergillus: global status in Europe and Asia. Ann N Y Acad Sci. 2012;1272:9-14.

142. Thompson 3rd GR et al. Development of caspofungin resistance following prolonged therapy for invasive candidiasis secondary to Candida glabrata infection. Antimicrob Agents Chemother. 2008;52(10):3783-5.

143. Fekkar A. et al., Emergence of echinocandin-resistant Candida spp. in a hospital setting: a consequence of 10 years of increasing use of antifungal therapy? Eur J Clin Microbiol Infect Dis, 2014. 33(9):1489-96

144. Beyda ND et al. FKS mutant Candida glabrata: risk factors and outcomes in patients with candidemia. Clin Infect Dis. 2014;59(6):819-25.

145. Blanchard E et al. Prior caspofungin exposure in patients with hematological malignancies is a risk factor for subsequent 
fungemia due to decreased susceptibility in Candida spp.: a casecontrol study in Paris, France. Antimicrob Agents Chemother. 2011;55(11):5358-61.

146. Fekkar A et al. Rapid emergence of echinocandin resistance during Candida kefyr fungemia treatment with caspofungin. Antimicrob Agents Chemother. 2013;57(5):2380-2.

147. Lewis J.S. 2nd, et al., Rapid emergence of echinocandin resistance in Candida glabrata resulting in clinical and microbiologic failure. Antimicrob Agents Chemother, 2013. 57(9): 4559-61

148. Ben-Ami $\mathrm{R}$ et al. The fitness and virulence cost of fks1 mutations causing echinocandin-resistance in Candida albicans. J Infect Dis. 2011;204(4):626-35.

149. Katiyar SK et al. Fks1 and Fks2 are functionally redundant but differentially regulated in Candida glabrata: implications for echinocandin resistance. Antimicrob Agents Chemother. 2012;56(12):6304-9. 